\title{
Associations between Body Height and Drinking Status with Diabetes in a Rural, Non-Overweight, Elderly, Japanese Male Population: The Nagasaki Islands Study
}

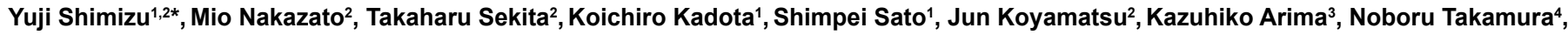
Kiyoshi Aoyagi ${ }^{3}$, Yosuke Kusano ${ }^{5}$ and Takahiro Maeda ${ }^{1,2}$

${ }^{1}$ Department of Community Medicine, Nagasaki University Graduate School of Biomedical Sciences, Nagasaki, Japan

${ }^{2}$ Department of Island and Community Medicine, Nagasaki University Graduate School of Biomedical Sciences, Nagasaki, Japan

${ }^{3}$ Department of Public Health, Nagasaki University Graduate School of Biomedical Sciences, Nagasaki, Japan

${ }^{4}$ Department of Global Health, Medicine and Welfare, Nagasaki University Graduate School of Biomedical Sciences, Atomic Bomb Disease Institute, Nagasaki University,

Nagasaki, Japan

${ }^{5}$ Department of Internal Medicine, Nagasaki National Hospital, Nagasaki, Japan

\begin{abstract}
Aim: A previous study of ours reported finding a positive association between height and hyperuricemia among non-overweight men $\left(\mathrm{BMl}<25 \mathrm{~kg} / \mathrm{m}^{2}\right)$. Although hyperuricemia is known to be also associated with diabetes, no published study has examined possible associations. Furthermore, while alcohol consumption is also reportedly associated with insulin resistance and risk of diabetes, there have been no reports on associations between height and diabetes which take drinking status into account.
\end{abstract}

Objective: We conducted a cross-sectional study to investigate the associations between height and diabetes in relation to drinking status among 787 non-overweight men aged 60-89 years undergoing a general health check-up.

Results: Of the study population consisting of 402 non-drinkers and 385 drinkers, 83 men (46 non-drinkers and 37 drinkers) were diagnosed with diabetes. Independent from classical cardiovascular risk factors, a significant positive association between height and diabetes was established for non-drinkers, whereas drinkers did not show any significant association. The cardiovascular risk factors-adjusted odds ratio (OR) and $95 \%$ confidence interval $(\mathrm{Cl})$ for diabetes for an increment of $1 \mathrm{SD}$ in height $(6.36 \mathrm{~cm})$ was $1.44(\mathrm{Cl}: 1.03-2.02)$ for non-drinkers and $1.01(\mathrm{Cl}$ : 0.67-1.53) for drinkers.

Conclusions: We were able to establish a significant positive association between height and diabetes for nonoverweight, elderly, Japanese, non-drinking men undergoing a health check-up. Since height indicates the influence of childhood social and physical condition childhood social and physical condition may contribute to the development of diabetes later in life.

Keywords: Height; Drinking status; Diabetes; Non-overweight; Elderly men

Abbreviations: AST: Aspartate Transaminase; GGT: $\gamma$ Glutamyltranspeptidase; $\mathrm{HbA}_{1 c}$ : Hemoglobin $\mathrm{A}_{1 \mathrm{c}}$; JDS: Japanese Diabetes Society; NGSP: National Glycohemoglobin Standardization Program; TG: Triglyceride

\section{Introduction}

A previous study of ours reported observing a significant positive association between height and hyperuricemia for non-overweight $\left(\mathrm{BMI}<25 \mathrm{~kg} / \mathrm{m}^{2}\right)$ men [1]. The reason for this association may be based on the assumption that height can be regarded as a surrogate marker of childhood social and physical condition while BMI may reflect primarily current physical condition [1-3], analysis of non-overweight subjects may elucidate a potential effect of height $[1,2]$. Furthermore, a Chinese study reported a positive association between plasma uric acid concentration and incidence of type 2 diabetes [4]. The Rancho Bernardo Study reported uric acid may be a useful predictor for diabetes in older adults with impaired fasting plasma glucose [5]. In another case, a control study, with 50 normal healthy individuals and 50 diabetes patients, reported that serum uric acid levels linearly increased with serum insulin levels in newly diagnosed diabetic patients [6]. A study investigating the association between height and diabetes among non-overweight subjects might indicate the influence of childhood social and physical condition on diabetes. However, no study has reported any association on those topics. Because women might not be suitable subjects for such an investigation since their height is negatively affected by vertebral compression fractures, which are mainly caused by postmenopausal osteoporosis [7], we limited our investigation to Japanese men. Other Japanese studies have reported that there may be a complicated association between drinking status and insulin resistance [8] as well as incidence of diabetes among Japanese men [9]. Since it is known that Japanese men are characterized by a high prevalence of drinking $[10,11]$, studies to investigate the association between height and diabetes should take drinking status into account. We therefore based our study on the hypothesis that height is positively associated

*Corresponding author: Yuji Shimizu, Department of Community Medicine, Nagasaki University Graduate School of Biomedical Sciences, Sakamoto 1-12-4, Nagasaki, 852-8523, Japan, Tel: +81-95-819-7578; Fax: +81-95-819-7189; E-mail: simizicyuu@yahoo.co.jp

Received January 09, 2014; Accepted February 19, 2014; Published February 24,2014

Citation: Shimizu Y, Nakazato M, Sekita T, Kadota K, Sato S, et al. (2014) Associations between Body Height and Drinking Status with Diabetes in a Rural Non-Overweight, Elderly, Japanese Male Population: The Nagasaki Islands Study. J Diabetes Metab 5: 339. doi:10.4172/2155-6156.1000339

Copyright: @ 2014 Shimizu Y, et al. This is an open-access article distributed under the terms of the Creative Commons Attribution License, which permits unrestricted use, distribution, and reproduction in any medium, provided the original author and source are credited. 
Citation: Shimizu Y, Nakazato M, Sekita T, Kadota K, Sato S, et al. (2014) Associations between Body Height and Drinking Status with Diabetes in a Rural, Non-Overweight, Elderly, Japanese Male Population: The Nagasaki Islands Study. J Diabetes Metab 5: 339. doi:10.4172/21556156.1000339

Page 2 of 6

with risk of diabetes for non-overweight, elderly, Japanese men, especially non-drinkers.

To validate this hypothesis, we conducted across-sectional study of a rural population of elderly, non-overweight, Japanese men who underwent general health check-ups between 2005 and 2012.

\section{Methods \\ Participants}

Written consent forms were available in Japanese to ensure comprehensive understanding of the study objectives, and informed consent was signed off on by the participants. This study was approved by the Ethics Committee for Use of Humans of Nagasaki University (Project Registration No.: 0501120073). The survey population covered 1,175 men aged 60 to 89 years, all residents of the western rural community of the Goto Islands, who participated in this study between 2005 and 2012. A total of 22 individuals with missing data and 366 overweight $\left(\mathrm{BMI} \geq 25 \mathrm{~kg} / \mathrm{m}^{2}\right)$ individuals were excluded because analysis among non-overweight subjects may elucidate a potential effect of height $[1,2]$ which is regarded as a surrogate marker of childhood social and physical condition [1-3,12,13], leaving 787 men for enrollment in this study (participation rate $=67.0 \%$ ). The mean age of the study population was 70.5 years ( $\pm 6.8 \mathrm{SD}$; range $60-89)$. In our study, the mean height values $(161.7 \mathrm{~cm}, \mathrm{SD}: 6.36)$ were almost the same as found by the Japan Public Health Center-based Prospective Study (JPHC Study) $(162.8 \mathrm{~cm}$, SD:6.43cm) of 15,564 Japanese men [12], and also the height quartiles $(<157.9 \mathrm{~cm}, 157.9-161.7 \mathrm{~cm}$, $161.8-165.7 \mathrm{~cm},>165.7 \mathrm{~cm})$ in our present study were similar to the CIRCS study $(<159 \mathrm{~cm}, 159-162 \mathrm{~cm}, 163-166 \mathrm{~cm},>166 \mathrm{~cm})$ of 4,567 Japanese men [2]. Another JPHC Study of 35,365 men reported that the prevalence of overweight participants (BMI $\geq 25 \mathrm{~kg} / \mathrm{m}^{2}$ ) was 28.4 $\%$ in their study [14], which is also similar to the prevalence in the present study (31.7\%). We therefore believed that our study included sufficient representativeness of the larger community, which might also be representative of the general Japanese population.

\section{Data collection and laboratory measurements}

Briefly, height in stocking feet and weight in light clothing were measured with an automatic body composition analyzer (BF-220; Tanita, Tokyo, Japan) when blood was drawn.

Systolic and diastolic blood pressures at rest were recorded using a blood pressure measuring device (HEM-907; Omron, Kyoto, Japan).

Fasting blood samples were obtained and the serum was separated and centrifuged after blood coagulation. Serum samples were also obtained in individual siliconized tubes. Serum triglycerides (TG), serum uric acid, and serum creatinine were measured using the enzyme method, serum HDL-cholesterol using the direct method, $\mathrm{HbA}_{1 \mathrm{c}}$ using the latex agglutination method, while serum Aspartate Aminotransferase (AST) was measured using the JASCC standardized method.

Trained interviewers obtained information on smoking status, drinking status, medical history, use of antihypertensive agents, lipidlowering medication use, and use of medication for diabetes mellitus. Hemoglobin $\mathrm{A}_{1 \mathrm{c}}\left(\mathrm{HbA}_{1 c}\right)$, as defined by the National Glycohemoglobin Standardization Program (NGSP), was calculated with the following equation, which was recently proposed by a working group of the Japanese Diabetes Society (JDS): $\mathrm{HbA}_{1 \mathrm{c}}$ (NGSP) $=\mathrm{HbA}_{1 \mathrm{c}}$ (JDS) $+0.4 \%$. Presence of diabetes was defined as $\mathrm{HbA}_{1 \mathrm{c}}$ (NGSP) $\geq 6.5 \%$, and/or initiation of glucose-lowering medication or insulin therapy.

\section{Statistical analysis}

We established height categories according to the quartiles of height for all non-overweight subjects.

Differences in age-adjusted mean values or prevalence of potential confounding factors were calculated per height quartile among all subjects using covariance or general linear models, and simple linear regression analysis was also performed between height and other variables. Logistic regression models were used for calculating odds ratios (OR) and 95\% confidence intervals (CI) to determine associations of diabetes with height.

Three different approaches were used to adjust for confounding factors. The first adjustment was only for age (Model 1), and the second used other possible confounding factors, namely: body mass index $\left(\mathrm{kg} / \mathrm{m}^{2}\right)$, smoking status (never been a smoker, former smoker, current smoker), alcohol intake (non-drinker, current light-to-moderate drinker (1-6 times/week) and current heavy drinker (every day)), systolic blood pressure ( $\mathrm{mmHg}$ ), antihypertensive medication use (no, yes), history of cardiovascular disease (no, yes) (Model2). Model3 also included lipid lowering medication use (no, yes), HDL-cholesterol (mg/ $\mathrm{dL})$, TG $(\mathrm{mg} / \mathrm{dL})$, AST $(\mathrm{IU} / \mathrm{L})$, serum creatinine $(\mathrm{mg} / \mathrm{dL})$ and serum uric acid $(\mathrm{mg} / \mathrm{dL})$. In addition, subjects were stratified by drinking status (non-drinker, drinker (current light-to-moderate drinker (1-6 times/week) and current heavy drinker (every day)) because alcohol consumption reportedly not only influences insulin resistance [8] but also incidence of diabetes in non-overweight men [9]. Furthermore, height may also be associated with drinking status [1-3,12,13], which might have a strong influence on height category and act as a confounding factor, and therefore we used the same height categories as for all subjects for both drinkers and non-drinkers.

All statistical analyses were performed with the SAS system for Windows (version 9.3; SAS Inc., Cary, NC). All p-values for statistical tests were two-tailed, and values of $<0.05$ were regarded as statistically significant.

\section{Results}

Of the 787 men taking part in the general health check-up program, 83 men were diagnosed with diabetes. The prevalence of current drinkers was $48.9 \%$.

Table 1 lists the characteristics of the total study population. The age-adjusted characteristics for this study population in relation to body height show that $\mathrm{HbA}_{1 \mathrm{c}}$ (NGSP), serum uric acid, and serum creatinine showed a significant positive association with body height. Also, whilst not significant, a positive association was observed between the prevalence of current drinkers and height $(\mathrm{p}=0.057)$. Simple correlation analysis of height revealed that there was a significant inverse association between age and height.

As shown in Table 2, even though a positive association was observed between height and diabetes for all subjects, it was not statistically significant.

To clarify the association between $\mathrm{HbA}_{1 \mathrm{c}}$ (NGSP) and drinking status, we performed additional analysis of participants who were not taking diabetic medication using the general linear model. In this analysis, we found a significant association between $\mathrm{HbA}_{1 \mathrm{c}}$ (NGSP) and drinking status. The age-adjusted $\mathrm{HbA}_{1 c}$ (NGSP) values (minimum mean square (minimum mean square error)) were: $5.6 \%(0.03 \%)$ for non-drinkers, $5.5 \%(0.04 \%)$ for current light-to-moderate drinkers (1-6 times/week), and $5.5 \%$ (0.04\%) for current heavy drinkers (every day) ( $\mathrm{P}$ for trend $=0.007$ ). 
Citation: Shimizu Y, Nakazato M, Sekita T, Kadota K, Sato S, et al. (2014) Associations between Body Height and Drinking Status with Diabetes in a Rural, Non-Overweight, Elderly, Japanese Male Population: The Nagasaki Islands Study. J Diabetes Metab 5: 339. doi:10.4172/21556156.1000339

Page 3 of 6

\begin{tabular}{|c|c|c|c|c|c|c|c|}
\hline & \multicolumn{5}{|c|}{ Age adjusted mean values and proportions by height } & \multirow{2}{*}{\multicolumn{2}{|c|}{$\begin{array}{l}\text { Simple correlation analysis of } \\
\text { height and other variables }\end{array}$}} \\
\hline & \multicolumn{5}{|c|}{ Height Quartile } & & \\
\hline & Q1 (low) & Q2 & Q3 & Q4 (High) & $P$ for trend & $\mathbf{r}$ & $\mathbf{p}$ \\
\hline Median Height level, cm & 154.6 & 159.7 & 163.9 & 169.0 & & & \\
\hline Number & 197 & 196 & 197 & 197 & & \multicolumn{2}{|c|}{2587} \\
\hline Age, years & $73.1 \pm 6.9$ & $71.5 \pm 7.0$ & $69.4 \pm 6.2$ & $68.0 \pm 6.2$ & $<0.001$ & -0.296 & $<0.001$ \\
\hline Systolic blood pressure, $\mathrm{mmHg}$ & 144 & 142 & 140 & 142 & 0.229 & -0.065 & 0.068 \\
\hline Diastolic blood pressure, $\mathrm{mmHg}$ & 83 & 83 & 82 & 84 & 0.254 & 0.086 & 0.015 \\
\hline Antihypertensive medication use, $\%$ & 29.1 & 35.7 & 29.0 & 34.7 & 0.312 & -0.025 & 0.488 \\
\hline Antidiabetic medication use, $\%$ & 5.3 & 5.5 & 8.7 & 8.9 & 0.367 & 0.047 & 0.187 \\
\hline Lipid lowering medication use, $\%$ & 5.4 & 6.1 & 3.6 & 7.3 & 0.462 & 0.017 & 0.634 \\
\hline History of cardiovascular disease, $\%$ & 9.0 & 14.0 & 13.1 & 11.2 & 0.436 & -0.016 & 0.649 \\
\hline Body mass Index, $\mathrm{Kg} / \mathrm{m}^{2}$ & 22.0 & 21.9 & 21.8 & 21.9 & 0.836 & -0.016 & 0.363 \\
\hline Current drinkers, $\%$ & 42.2 & 46.2 & 51.9 & 55.3 & 0.057 & 0.140 & $<0.001$ \\
\hline Current smokers, \% & 22 & 25 & 19 & 22 & 0.635 & 0.009 & 0.791 \\
\hline $\mathrm{HbA}_{1 \mathrm{c}}$ (NGSP), \% & 5.7 & 5.9 & 5.9 & 6.2 & 0.012 & 0.012 & 0.730 \\
\hline Serum HDL-Cholesterol, mg/dL & 56 & 56 & 58 & 55 & 0.093 & 0.009 & 0.795 \\
\hline Serum triglycerides (TG), mg/dL & 112 & 105 & 107 & 112 & 0.707 & 0.038 & 0.278 \\
\hline Serum aspartate aminotranferase (AST) IU/L & 25 & 25 & 25 & 24 & 0.384 & -0.071 & 0.045 \\
\hline Serum uric acid, mg/dL & 5.7 & 5.9 & 5.9 & 6.2 & 0.012 & 0.128 & $<0.001$ \\
\hline Serum creatinine, $\mathrm{mg} / \mathrm{dL}$ & 0.87 & 0.89 & 0.91 & 0.97 & 0.002 & 0.119 & $<0.001$ \\
\hline
\end{tabular}

Age: Mean \pm Standard Deviation, P: P value, NGSP: National Glycohemoglobin Standardization Program, Height quartile: $<157.9 \mathrm{~cm}, 157.9-161.7 \mathrm{~cm}, 161.8-165.7 \mathrm{~cm}$ and $>165.7 \mathrm{~cm}$.

Table 1: Characteristics of total study population.

\begin{tabular}{|l|c|c|c|c|c|}
\hline & \multicolumn{3}{|c|}{ Height Quartile } & \multicolumn{2}{|c|}{$\begin{array}{l}\text { Per 1 SD increment } \\
\text { in height (6.36 cm) }\end{array}$} \\
\hline All subjects & Q1 (low) & Q2 & & \\
\hline Number & 197 & 196 & 197 & \\
\hline $\begin{array}{l}\text { No. of cases } \\
\text { (percentage) }\end{array}$ & $19(9.6)$ & $17(8.7)$ & $23(11.7)$ & $24(12.2)$ \\
\hline Model 1 & 1.00 & $0.91(0.46-1.81)$ & $1.29(0.67-2.49)$ & $1.38(0.71-2.68)$ & 0.223 \\
\hline Model 2 & 1.00 & $0.89(0.44-1.78)$ & $1.31(0.68-2.55)$ & $1.41(0.72-2.77)$ & $1.14(0.90-1.44)$ \\
\hline Model 3 & 1.00 & $0.92(0.46-1.84)$ & $1.35(0.69-2.64)$ & $1.62(0.82-3.20)$ & 0.193 \\
\hline
\end{tabular}

Model 1: Adjusted for age, Model 2: Age + other CVD risk factors (systolic blood pressure, antihypertensive medication use, body mass index, smoking, alcohol intake, and history of cardiovascular disease), Model 3: Model 2+serum HDL-cholesterol, serum triglycerides (TG) lipid-lowering medication use, serum aspartate aminotranferase (AST), serum uric acid, and serum creatinine, High quartiles: $<157.9 \mathrm{~cm}, 157.9-161.7 \mathrm{~cm}, 161.8-165.7 \mathrm{~cm}$ and $>165.7 \mathrm{~cm}$.

Table 2: Odds Ratios (OR) and $95 \%$ confidence Intervals $(\mathrm{Cl})$ for Diabetes in relation to height for all subjects.

We conducted further investigations to evaluate height in relation to drinking status using the general linear model. Furthermore, we found that height was also significantly associated with drinking status. The age-adjusted values of height (minimum mean square (minimum mean square error)) were: $161.0 \mathrm{~cm}(0.3 \mathrm{~cm})$ for non-drinkers, 162.0 $\mathrm{cm}(0.4 \mathrm{~cm})$ for current light-to-moderate drinkers (1-6 times/week), and $162.6 \mathrm{~cm}(0.4 \mathrm{~cm})$ for current heavy drinkers (every day) ( $\mathrm{P}$ for trend $=0.009$ ). Since drinking status was established after childhood and we also found height was significantly associated with drinking status, this significant association is not caused by alcohol consumption, although height might affect drinking status in later life. This means height might partly indicate a capacity for alcohol tolerance. Therefore, drinking status-specific height values (mean: $160.9 \mathrm{~cm}, \mathrm{SD}: 6.6 \mathrm{~cm}$ for non-drinkers, and mean: $162.6 \mathrm{~cm}, \mathrm{SD}: 5.9 \mathrm{~cm}$ for drinkers) might have a confounding effect on the influence of height on diabetes. Thus, we used the same height categories, which were categorized among all subjects, to investigate the association between height and diabetes in relation to drinking status.
Table 3 lists associations between height and diabetes stratified by drinking status, showing a significant association between height and diabetes for non-drinkers but no such significant association for drinkers. The fully-adjusted odds ratio (OR) and 95\% confidence interval (CI) for diabetes for an increment of $1 \mathrm{SD}$ in height $(6.36 \mathrm{~cm})$ were 1.44 (1.03-2.02) for non-drinkers and 1.01 (0.67-1.53) for drinkers.

\section{Discussion}

The main finding of the present study was that height is associated with diabetes for non-overweight, elderly, non-drinking Japanese men undergoing health check-ups in the Goto Islands. Since height indicates the influence of childhood social and physical condition, childhood social and physical condition may contribute to the development of diabetes later in life.

The impairment of glucose tolerance with age was first recognized by Spence [15] in subjects over 60 -years old. More recent studies have indicated that muscle is the principal site of development of a significant impairment with age of peripheral glucose uptake [16-19]. Aging is 
Citation: Shimizu Y, Nakazato M, Sekita T, Kadota K, Sato S, et al. (2014) Associations between Body Height and Drinking Status with Diabetes in a Rural, Non-Overweight, Elderly, Japanese Male Population: The Nagasaki Islands Study. J Diabetes Metab 5: 339. doi:10.4172/21556156.1000339

Page 4 of 6

\begin{tabular}{|c|c|c|c|c|c|c|}
\hline & \multicolumn{5}{|c|}{ Height Quartile } & \multirow[b]{2}{*}{$\begin{array}{l}\text { Per } 1 \text { SD increment } \\
\text { in height } 6.36 \mathrm{~cm}\end{array}$} \\
\hline & Q1 (low) & Q2 & Q3 & Q4 (High) & $P$ for trend & \\
\hline \multicolumn{7}{|l|}{ Non-drinkers } \\
\hline Number & 118 & 107 & 93 & 84 & & \\
\hline No. of cases (percentage) & $10(8.5)$ & $12(11.2)$ & $10(10.8)$ & $14(16.7)$ & & \\
\hline Model 1 & 1.00 & $1.43(0.59-3.49)$ & $1.56(0.60-4.01)$ & $2.82(1.13-7.05)$ & 0.032 & $1.40(1.01-1.93)$ \\
\hline Model 2 & 1.00 & $1.33(0.54-3.29)$ & $1.44(0.54-3.79)$ & $2.76(1.08-7.01)$ & 0.041 & $1.38(1.00-1.93)$ \\
\hline Model 3 & 1.00 & $1.34(0.53-3.34)$ & $1.39(0.52-3.73)$ & $3.02(1.17-7.82)$ & 0.032 & $1.44(1.03-2.02)$ \\
\hline \multicolumn{7}{|l|}{ Drinkers } \\
\hline Number & 79 & 89 & 104 & 113 & & \\
\hline No. of cases (percentage) & $9(11.4)$ & $5(5.6)$ & $13(12.5)$ & $10(8.8)$ & & \\
\hline Model 1 & 1.00 & $0.43(0.14-1.34)$ & $0.98(0.39-2.47)$ & $0.64(0.24-1.71)$ & 0.736 & $0.91(0.63-1.34)$ \\
\hline Model 2 & 1.00 & $0.43(0.14-1.38)$ & $0.98(0.38-2.50)$ & $0.65(0.24-1.76)$ & 0.750 & $0.92(0.63-1.36)$ \\
\hline Model 3 & 1.00 & $0.41(0.13-1.37)$ & $1.05(0.40-2.75)$ & $0.74(0.26-2.12)$ & 0.977 & $1.01(0.67-1.53)$ \\
\hline
\end{tabular}

Model 1: Adjusted for age, Model 2: Age + other CVD risk factors (systolic blood pressure, antihypertensive medication use, body mass index, smoking, alcohol intake, and history of cardiovascular disease), Model 3: Model 2+serum HDL-cholesterol, serum triglycerides (TG) lipid-lowering medication use, serum aspartate aminotranferase (AST), serum uric acid, and serum creatinine, High quartiles: $<157.9 \mathrm{~cm}, 157.9-161.7 \mathrm{~cm}, 161.8-165.7 \mathrm{~cm}$ and $>165.7 \mathrm{~cm}$.

Table 3: Odds Ratios (OR) and 95\% confidence Intervals $(\mathrm{Cl})$ for Diabetes in relation to height stratified by drinking status.

associated with reduced neuromuscular function [20]; in particular, it has been suggested that changes in the number of cells and levels of neuromuscular systems occur with advancing age [21-25]. Moreover, in present study taller people may suffer a much greater reduction in total muscle mass with advancing age than do shorter people. In fact, previous cross-sectional studies in North America and Europe have described the sarcopenic index, which is used to diagnose the age-associated loss of skeletal muscle mass and function, as: muscle mass $\div$ (height $)^{2}$ $[26,27]$. In the present study, concentration of serum creatinine, which is a well=known factor that is positively associated with muscle volume, also showed a significant positive association with height. As far as non-overweight, elderly men are concerned, taller individuals may therefore be at a higher risk of impaired glucose tolerance than their shorter counter parts. To prevent incidence of diabetes, taller elderly individuals should make more active lifestyle changes to adapt to aging than their shorter counterparts.

Since previous studies had reported a positive association between blood concentration of uric acid and diabetes [4-6], and a previous study of ours had reported a positive association between height and hyperuricemia (serum uric acid $>7.0 \mathrm{mg} / \mathrm{dL}$ ) [1], we conducted further investigations. As a result, we found no significant associations between diabetes and hyperuricemia (serum uric acid $>7.0 \mathrm{mg} / \mathrm{dL}$ ). The age-adjusted ORs of diabetes for hyperuricemia were: $0.84(0.48$ 1.47, $\mathrm{P}=0.544)$ for all subjects, $1.05(0.48-2.28, \mathrm{P}=0.911)$ for nondrinkers and $0.70(0.31-1.58, \mathrm{P}=0.386)$ for drinkers. We also observed a significant association between serum uric acid and serum creatinine; the simple linear regression analysis showed; $r=0.35(\mathrm{P}=<0.001)$ for all subjects, $r=0.37(\mathrm{P}<0.001)$ for non-drinkers, and $\mathrm{r}=0.34(\mathrm{P}<0.001)$ for drinkers. Both serum concentration of uric acid and serum creatinine are well=known factors that are strongly influenced by renal function, and short stature maybe correlated with renal dysfunction $[28,29]$. The association of hyperuricemia with diabetes might be confounded by renal impairment that could potentially be more frequent among participants of shorter stature. Further investigation using data on precise renal function is necessary.

However, it is not yet clear why no positive association between height and diabetes was observed among drinkers. A previous cross-sectional study of 1,902 asymptomatic Japanese men reported detecting an inverse association between alcohol consumption and insulin resistance, independent of central obesity, metabolic profiles, or fatty liver disease [8]. On the other hand, another previous Japanese prospective study of 12,913 men showed moderate-to-high alcohol consumption was positively associated with incidence of diabetes for BMI $\leq 22 \mathrm{~kg} / \mathrm{m}^{2}$ while no significant association was observed for 22 $\mathrm{kg} / \mathrm{m}^{2}<\mathrm{BMI} \leq 25 \mathrm{~kg} / \mathrm{m}^{2}$ [9]. These studies clearly point to the influence of alcohol consumption on risk of diabetes. Our follow-up study, which excluded potential participants who were taking anti-diabetic medication, showed a significant inverse association between $\mathrm{HbA}_{1 \mathrm{c}}$ (NGSP) and drinking status. Even though we were able to conclude that $\mathrm{HbA}_{1 \mathrm{c}}$ (NGSP) was influenced by drinking status, a larger BMIstratified study is necessary to clarify the influence of drinking status on $\mathrm{HbA}_{1 \mathrm{c}}$ (NGSP). In addition to those complicated reports, height may also be associated with prevalence of drinking status, since in our study, height showed a non-significant positive association with current drinking status, as was also reported by previous studies $[1-3,12,13]$, and in the follow-up study, a significant association between height and drinking status (non-drinker, drinker current light-to-moderate drinker (1-6 times/week) and current heavy drinker (every day)) was observed. Therefore, alcohol may act as a confounding factor for the association between height and diabetes.

Potential limitations of this study warrant consideration. Since we did not have access to data regarding quantities of alcohol consumption, we classified current drinking status only in terms of frequency of drinking per week, which may have also confounded the association between height and diabetes among current drinkers. Also, because we did not have enough data to conduct a stratified analysis by alcohol consumption for current drinkers, we could not determine the exact influence of alcohol consumption. However, we observed a significant positive association between height and diabetes among non-drinkers. Since drinking status is a known factor that is determined after childhood when the height has already been determined, we therefore thought the association between height and diabetes among drinkers is only due to the confounding effect of drinking status. Even though we did not observe a significant association between height and diabetes among drinkers, such associations may be exhibited by drinkers in addition to non-drinkers. Further studies with larger numbers of participants will therefore be necessary. Since data on exercise and food intake was not available, we could not make adjustments for the influence of those factors as in our previous study [30,31]. However, in our present 
Citation: Shimizu Y, Nakazato M, Sekita T, Kadota K, Sato S, et al. (2014) Associations between Body Height and Drinking Status with Diabetes in a Rural, Non-Overweight, Elderly, Japanese Male Population: The Nagasaki Islands Study. J Diabetes Metab 5: 339. doi:10.4172/21556156.1000339

study, the multivariable adjusted model including BMI values showed a significant association. As in previous studies [1-3,10,11,30,31], our survey population consisted of men undergoing a general health checkup. The influence of selection bias was underlying. Since a previous study reported that the mean height for the age range 30-39was lower for men born between 1936 and $1945(163.8 \mathrm{~cm})$ than for those born between 1961 to $1970(170.6 \mathrm{~cm})$ [13], this difference might be due to a change in diet and food consumption from the period during World War II to the post-war period. Since Q1 was the oldest age group, it was not unexpected that diabetes was most prevalent in Q1 and less prevalent in Q4, which was the youngest age group. However, our present study showed height is positively associated with diabetes among nondrinking, non-overweight men. Furthermore, the associations between height and diabetes were the same even when we limited the analysis to older subjects who were born in or before 1945; the fully-adjusted ORs of diabetes for an increment of 1SD in height were: 1.27 (0.98-1.66) for all subjects, 1.52 (1.06-2.16) for non-drinkers and 0.98 (0.62-1.53) for drinkers. Furthermore, because we do not have data on distinct diabetes duration and progression, type of diabetes and duration of medication, we could not take account of those factors in the present study. Finally, we could not establish any causal relationships because this study was cross-sectional. However, since height was regarded as a surrogate marker of childhood social and physical condition [1$3,12,13$ ] and the target population in the present study was elderly men, we believe this investigation has the characteristics of a prospective study to a certain degree. Further prospective population based studies are needed to eliminate the possibility of a causal relationship between height and diabetes.

In conclusion, we determined that height is independently and positively associated with diabetes for non-overweight, elderly, Japanese non-drinking men undergoing health check-ups in the Goto Islands. Since height indicates the influence of childhood social and physical condition, childhood social and physical condition may contribute to the development of diabetes later in life.

\section{Acknowledgements}

This work was supported by Grants-in-Aid for Scientific Research from the Japan Society for the Promotion of Science (No. 22370090). The authors declare that they have no conflicting interests.

\section{References}

1. Shimizu Y, Nakazato M, Sekita T, Kadota K, Arima K, et al. (2013) Relationships of adult body height and BMI status to hyperuricemia in general Japanese male population: The Nagasaki Islands Study. Acta Med Nagasaki 58: 57-62.

2. Shimizu Y, Imano H, Ohira T, Kitamura A, Kiyama M, et al. (2013) Adult Height and Body Mass Index in Relation to Risk of Total Stroke and its Subtypes: The Circulatory Risk in Communities Study. J Stroke Cerebrovasc Dis (in press).

3. Shimizu Y, Nakazato M, Sekita T, Kadota K, Arima K, et al. (2013) Relationship between adult height and body weight and risk of carotid atherosclerosis assessed in terms of carotid intima-media thickness: The Nagasaki Island study. J Physiol Anthropol 32: 19.

4. Chien KL, Chen MF, Hsu HC, Chang WT, Su TC, et al. (2008) Plasma uric acid and the risk of type 2 diabetes in a Chinese community. Clin Chem 54: 310-316.

5. Kramer CK, von MühlenD, Jassal SK, Barrett-Connor E (2009) Serum uric acid levels improve prediction of incident type 2 diabetes in individuals with impaired fasting glucose: the Rancho Bernardo Study. Diabetes Care 32: 1272-1273.

6. Gill A, Kukreja S, Malhotra N, Chhabra N (2013) Correlation of the serum insulin and the serum uric Acid levels with the glycated haemoglobin levels in the patients of type 2 diabetes mellitus. J Clin Diagn Res 7: 1295-1297.

7. Nasto LA, Fusco A, Colangelo D, Mormando M, Di Giacomo G, et al. (2012) Clinical predictors of vertebral osteoporotic fractures in post-menopausal women: a cross-sectional analysis. Eur Rev Med Pharmacol Sci 16: $1227-$ 1234.
8. Gunji T, Matsuhashi N, Sato H, lijima K, Fujibayashi K, et al. (2011) Alcohol consumption is inversely correlated with insulin resistance, independent of metabolic syndrome factors and fatty liver diseases. J Clin Gastroenterol 45 : 808-813.

9. Waki K, Noda M, Sasaki S, Matsumura Y, Takahashi Y, et al. JPHC Study Group (2005) Alcohol consumption and other risk factors for self-reported diabetes among middle-aged Japanese: a population-based prospective study in the JPHC study cohort I. Diabet Med 22: 323-331.

10. Shimizu Y, Imano H, Ohira T, Kitamura A, Kiyama M, et al. (2013) Alkaline phosphatase and risk of stroke among Japanese: the Circulatory Risk in Communities Study (CIRCS). J Stroke Cerebrovasc Dis 22: 1046-1055.

11. Shimizu Y, Maeda K, Imano H, Ohira T, Kitamura A, et al. (2011) Chronic kidney disease and drinking status in relation to risks of stroke and its subtypes: the Circulatory Risk in Communities Study (CIRCS). Stroke 42: 2531-2537.

12. Honjo K, Iso $\mathrm{H}$, Inoue M, Tsugane S (2011) Adult height and the risk of cardiovascular disease among middle aged men and women in Japan. Eur $\mathrm{J}$ Epidemiol 26: 13-21.

13. Hozawa A, Murakami Y, Okamura T, Kadowaki T, Nakamura K, et al. (2007) Relation of adult height with stroke mortality in Japan: NIPPON DATA80. Stroke 38: $22-26$.

14. Yoshida M, Inoue M, Iwasaki M, Tsugane S; JPHC Study Group (2010) Association of body mass index with risk of age-related cataracts in a middleaged Japanese population: the JPHC Study. Environ Health Prev Med 15: $367-$ 373.

15. Spence JW (1921) Some observations on sugar tolerance with special reference to variations found at different ages. QJM 14: 314-326.

16. Jackson RA, Blix PM, Matthews JA, Hamling JB, Din BM, et al. (1982) Influence of ageing on glucose homeostasis. J Clin Endocrinol Metab 55: 840-848.

17. Defronzo RA (1979) Glucose intolerance and aging: evidence for tissue insensitivity to insulin. Diabetes 28: 1095-1101.

18. Robert JJ, Cummins JC, Wolfe RR, Durkot M, Matthews DE, et al. (1982) Quantitative aspects of glucose production and metabolism in healthy elderly subjects. Diabetes 31: 203-211.

19. Fink RI, Kolterman OG, Griffin J, Olefsky JM (1983) Mechanisms of insulin resistance in aging. J Clin Invest 71: 1523-1535.

20. Manini TM, Clark BC (2012) Dynapenia and aging: an update. J Gerontol A Bio Sci Med Sci 67: 28-40.

21. Russ DW, Gregg-Cornell K, Conaway MJ, Clark BC (2012) Evolving concepts on the age-related changes in "muscle quality". J Cachexia Sarcopenia Muscle 3: 95-109.

22. Clark BC, Taylor JL (2011) Age-related changes in motor cortical properties and voluntary activation of skeletal muscle. Curr Aging Sci 4: 192-199.

23. Delbono O (2011) Expression and regulation of excitation-contraction coupling proteins in aging skeletal muscle. Curr Aging Sci 4: 248-259.

24. Deschenes MR (2011) Motor unit and neuromuscular junction remodeling with aging. Curr Aging Sci 4: 209-220.

25. Fry CS, Rasmussen BB (2011) Skeletal muscle protein balance and metabolism in the elderly. Curr Aging Sci 4: 260-268.

26. Cruz-Jentoft AJ, Baeyens JP, Bauer JM, Boirie Y, Cederholm T, et al. (2010) Sarcopenia: European consensus on definition and diagnosis: Report of the European Working Group on Sarcopenia in Older People. Age Ageing 39: 412423.

27. Fielding RA, Vellas B, Evans WJ, Bhasin S, Morley JE, et al. (2011) Sarcopenia: an undiagnosed condition in older adults. Current contentious definition: prevalence, etiology, and consequences. International working group on sarcopenia. J Am Med Dir Assoc 12: 249-256.

8. Sørensen HT, Sabroe S, Rothman KJ, Gillman M, Steffensen FH, et al. (1999) Birth weight and length as predictors for adult height. Am J Epidemiol 149 726-729.

29. Hughson M, Farris AB 3rd, Douglas-Denton R, Hoy WE, Bertram JF (2003) Glomerular number and size in autopsy kidneys: the relationship to birth weight. Kidney Int 63: 2113-2122. 
Citation: Shimizu Y, Nakazato M, Sekita T, Kadota K, Sato S, et al. (2014) Associations between Body Height and Drinking Status with Diabetes in a Rural, Non-Overweight, Elderly, Japanese Male Population: The Nagasaki Islands Study. J Diabetes Metab 5: 339. doi:10.4172/21556156.1000339

Page 6 of 6

30. Shimizu Y, Nakazato M, Sekita T, Kadota K, Yamasaki H, et al. (2013)Association of arterial stiffness and diabetes with triglycerides-to-HDL cholesterol ratio for Japanese men: the Nagasaki Islands Study. Atherosclerosis 228: 491-495
31. Shimizu Y, Nakazato M, Sekita T, Kadota K, Sato S, et al. (2013) Body mass index and triglyceride-to-HDL-cholesterol ratio in relation to risk of diabetes: The Nagasaki Islands study. Acta Med Nagasaki 58: 85-91. 